\title{
Liver transplantation for irresectable colorectal liver metastases: still a contraindication?
}

\author{
Astrid Herrero $^{1}$, Silvio Nadalin ${ }^{2}$, Fabrizio Panaro ${ }^{1}$ \\ ${ }^{1}$ Department of General and Liver Transplant Surgery, University Hospital Montpellier, Hôpital Saint Eloi, Montpellier, France; ${ }^{2}$ Department of \\ General, Visceral and Transplant Surgery, University Hospital Tübingen, Tübingen, Germany \\ Correspondence to: Fabrizio Panaro, MD, PhD. Department of Surgery, Liver \& Pancreas Transplant Unit, Montpellier University Hospital, College \\ of Medicine, 80, Avenue Augustin Fliche, 34295 Montpellier Cedex 5, France. Email: f-panaro@chu-montpellier.fr.
}

Submitted Aug 07, 2018. Accepted for publication Aug 15, 2018.

doi: $10.21037 /$ hbsn.2018.08.04

View this article at: http://dx.doi.org/10.21037/hbsn.2018.08.04

Liver transplantation (LT) for irresectable colorectal cancer liver metastases (i-CRLM) has been considered up to now an absolute contraindication due to unfavorable outcomes, scarcity of grafts and ethical considerations. Recently, promising results of the Norwegian SECA trial raise the question of the utility of LT for i-CRLM with a 5 -year survival rate of $60 \%$ (1). However, some concerns should be considered as to oncological selection criteria (when to perform the transplantation?) and the source of liver grafts: deceased donor at cost of recipient listed for standard indication or living donor in a population with a higher risk of recurrence.

Despite progress in neoadjuvant therapy including epidermal growth factor receptor (EGFR) antibodies and aggressive surgical treatment, only $10 \%$ to $20 \%$ of CRLM are resectable with a 5 -year survival about $30 \%$ to $40 \%$ (2). Most patients with CRLM have irresectable disease and standard treatment is palliative chemotherapy with a poor 5 -year survival definitely less than $10 \%$ (3). To improve survival, new therapeutic strategies are needed in this large population. LT is an attractive option with a R0 resection in patients without portal hypertension, coagulopathy or renal dysfunction. Although instead of whereas the surgical risk is lower compared to cirrhotic patients, the major risk of this strategy is the risk of recurrence, which may be increased by immunosuppression, and thus the futility of LT. However, evolving management of hepatocellular carcinoma including LT is an example of the great benefit on survival outcomes for selected patients using adapted graft allocation tools. LT for malignancy has become a major indication in the last decade, and accounts for about $30 \%$ of patients with LT (4). The development of multidisciplinary teams allows multimodal treatments including chemotherapy, targeted therapy, interventional radiology, radiotherapy, surgical resection and LT. These advances open a new Era in transplant oncology. Indications of LT for malignancy can be expanded from the moment the rules of fairness are respected. In addition, we are witnessing a change in the epidemiology of liver disease as a result of successful HCV therapy. Eradication of hepatitis $\mathrm{C}$, which accounted for one third of the transplanted, is foreseen for 2025 (5). If this estimate confirmed, more liver grafts would be available in the next future for standard and new/extended indications.

The surgical and medical advances in LT the last 2 decades bring up to distinguish 2 study periods to analyze the indication of LT for malignancy, especially for CRLM. The development of multidisciplinary team after to 2000 define the new Era $(6,7)$. The first results published about CRLM and LT in the 1990's were very poor with a 5 -year survival rate of $18 \%$. A total of 55 patients were registered by the European LT Registry (8) including the first series performed at the University of Vienna. These transplantations were performed before 1995 without selection criteria, with variable immunosuppression protocols and with a high rate of postoperative mortality due to surgical risks.

In 2006, the group from the Oslo University Hospital conducted an open, prospective pilot study to assess the survival in i-CRLM after LT (SECA Trial) including 21 
patients. Norway has a particularity with more donors than potential recipients, and the median waiting time for LT is less than 1 month. The inclusion criteria for this trial were R0 primary colorectal resection; at least 6 weeks of one or more chemotherapy agents received for metastatic disease; irresectable liver metastases; no extrahepatic disease; and Eastern Cooperative Oncology Group (ECOG) performance status $0-1$. The median follow-up time was 27 months and the 1-, 3-, and 5 -year overall survival (OS) was $95 \%, 68 \%$, and $60 \%$, respectively. Recurrence rate was very high, about $90 \%$, with a median disease-free survival (DFS) of 10 months, and $30 \%$ of patients deceased with disseminated metastases. No preoperative or adjuvant chemotherapy standardized protocol was administrated in this study and the posttransplant immunosuppressive protocol included sirolimus. The main limitations of this study were the small sample and the relative short follow-up time.

Patterns of recurrence after LT for i-CRLM were investigated (9). In the majority of cases, the lungs were the first single site of recurrence, having slow growth and could be resected. No patients had liver-only disease and in none was the liver the first recurrence site. From the SECA 1 trial four factors emerged as predictive of poor survival: (I) diameter of the largest tumor above $55 \mathrm{~mm}$; (II) carcinoembryonic antigen level higher than 80 (normal value: $<2.5 \mathrm{ng} / \mathrm{mL}$ ); (III) progressive disease on chemotherapy; (IV) time from resection of the primary tumor to transplant less than 2 years.

These factors, with the exclusion of the tumor size, were confirmed by Toso et al. (European consortium series of 12 patients) in 2017 (10).

The results of LT for i-CRLM in the last decade were better than in the previous era with a 5-year survival around $50 \%$, thanks to the development of effective chemotherapy regimens and to the dramatic improvements in LT surgical technique and perioperative recipient care.

From here on in, it is necessary to define selection criteria for patients who can benefit from LT and then standardize the procedure to consolidate these encouraging results. A new randomized controlled trial was initiated in Oslo in 2011 (SECA 2 study) to assess the OS between patients undergoing LT or liver resection. In this protocol, more stringent selection criteria have been used and the results will be published in 2027 .

Improvement in preoperative imaging studies (CT,
FDG-PET with metabolic parameters: metabolic tumor volume and total lesion glycolysis) could be the crucial factor in selection of the best candidates for LT (11).

However, a fundamental limitation is the scarcity of the liver grafts. This is the major ethical barrier for this kind of indication. Several ways are discussed to expand the pool of grafts using split or extended criteria liver donors. In this context a new surgical technique has been recently proposed by the Oslo group but it is a technical challenge. This new approach called RAPID (resection and partial liver segment $2 / 3$ transplantation with delayed total hepatectomy) has been described recently (12). The protocol consists in: (I) native left hepatectomy; (II) auxiliary orthotopic transplantation of the left lateral lobe as a split graft from deceased donor; (III) left lobe hypertrophy (portal inflow modulation in 2-3 weeks); (IV) total native hepatectomy delayed until the transplanted graft has reached sufficient volume. The results are promising but it is too early to conclude. In this context, left lateral living donor LT may be the ideal solution. This year Königsrainer et al. published the first case of left lateral living donor auxiliary partial orthotopic liver transplant (APOLT) and 2 stage hepatectomy according to the RAPID concept (LD-RAPID concept) (13).

Today, 4 clinical trials and 2 cohort studies on LT for CRLM are registered on clinical trial.gov and are summarized in Table 1. International registry is necessary. The RAPID trial will assess the safety and benefit of this technique in transplanted patients receiving second hepatectomy within 4 weeks of segment $2 / 3$ implantation and OS. The study is planned to be achieved in 2028. The TRANSMET trial from France is recruiting patients into a randomized open label trial. Patients with i-CRLM will be randomized to receive standard of care chemotherapy or LT plus chemotherapy. The main outcome is 3 - and 5 -year DFS/progression-free survival. The results are planned to be published in 2027 .

In conclusion, the current status suggests that it is possible to obtain prolonged DFS and OS for patients with irresectable CRLM. However, synonymous patient selection is mandatory. Several questions remain unanswered. The transplant community is very interested in this problem and has organized many trials which are under way. The expected results within the next 10 years will probably drastically change the management of CRLM and will redefine the place of the living donor in LT. 


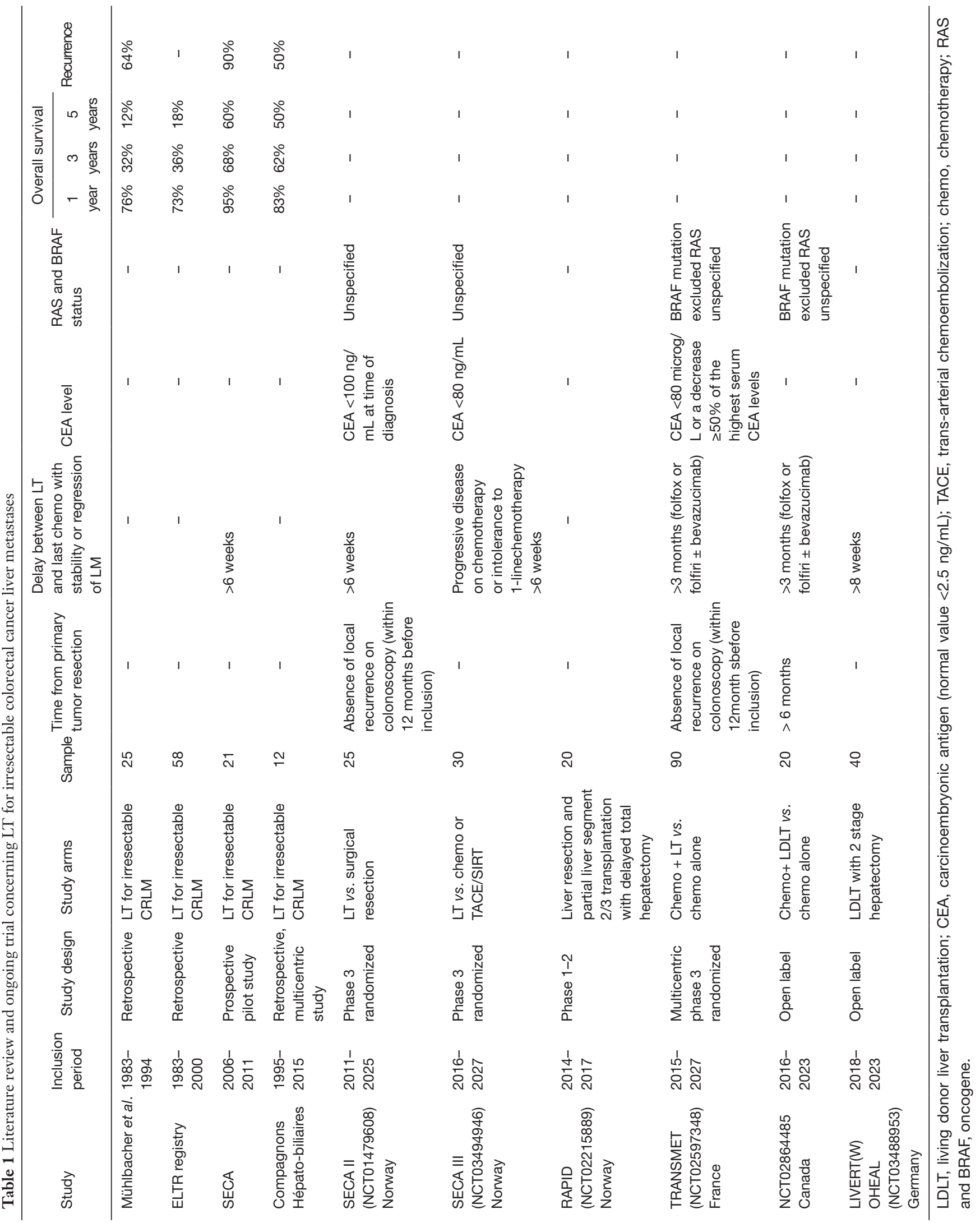




\section{Acknowledgements}

None.

\section{Footnote}

Conflicts of Interest: The authors have no conflicts of interest to declare.

\section{References}

1. Hagness M, Foss A, Line PD, et al. Liver transplantation for nonresectable liver metastases from colorectal cancer. Ann Surg 2013;257:800-6.

2. Kanas GP, Taylor A, Primrose JN, et al. survival after liver resection in metastatic colorectal cancer: review and metaanalysis of prognostic factors. Clin Epidemiol 2012;4:283-301.

3. Dueland S, Guren TK, Hagness M, et al. Chemotherapy or liver transplantation for nonresectable liver metastases from colorectal cancer? Ann Surg 2015;261:956-60.

4. Llovet JM, Zucman-Rossi J, Pikarsky E, et al. Hepatocellular carcinoma. Nat Rev Dis Primers 2016;2:16018.

5. Vermehren J, Park JS, Jacobson I, et al. Challenges and perspectives of direct antivirals for the treatment of hepatitis C virus infection. J Hepatol 2018. [Epub ahead of print].

6. Hibi T, Itano O, Shinoda $M$, et al. Liver transplantation for hepatobiliary malignancies: a new era of "Transplant

Cite this article as: Herrero A, Nadalin S, Panaro F. Liver transplantation for irresectable colorectal liver metastases: still a contraindication? HepatoBiliary Surg Nutr 2018;7(6):475-478. doi: 10.21037/hbsn.2018.08.04
Oncology" has begun. Surg Today 2017;47:403-15.

7. Gorgen A, Muaddi H, Zhang $W$ et al. The New Era of Transplant Oncology: liver Transplantation for Nonresectable colorectal cancer liver metastases. Can J Gastroenterol Hepatol 2018;2018:9531925.

8. Hoti E, Adam R. Liver transplantation for primary and metastatic liver cancers. Transpl Int 2008;21:1107-17.

9. Hagness M, Foss A, Egge TS, et al. Patterns of recurrence after liver transplantation for nonresectable liver metastases from colorectal cancer. Ann Surg Oncol 2014;21:1323-9.

10. Toso C, Pinto Marques H, Andres A, et al. Liver transplantation for colorectal liver metastasis: Survival without recurrence can be achieved. Liver Transpl 2017;23:1073-6.

11. Grut H, Revheim ME, Line PD, et al. Importance of 18F-FDG PET/CT to select patients with nonresectable colorectal liver metastases for liver transplantation. Nucl Med Commun 2018;39:621-7.

12. Line PD, Hagness M, Berstad AE, et al. A Novel Concept for Partial Liver Transplantation in Nonresectable Colorectal Liver Metastases: The RAPID Concept. Ann Surg 2015;262:e5-9.

13. Königsrainer A, Templin S, Capobianco I, et al. Paradigm Shift in the Management of Irresectable Colorectal Liver Metastases: Living Donor Auxiliary Partial Orthotopic Liver Transplantation in Combination With Two-stage Hepatectomy (LD-RAPID). Ann Surg 2018. [Epub ahead of print]. 